Instrumental A chievements

\title{
Crystal and Molecular Structures of the Methyl Ester of the 3-Formyl-4,6- dihydroxy-5-isopropyl-2-methylbenzoic Acid: a Synthetic Intermediate to the Espintanol
}

\author{
Adrián Covarrubias-ZúñIga, ${ }^{\dagger}$ Noé ZÚÑIGa-Villarreal, ${ }^{\dagger}$ and Rubén A. Toscano \\ Instituto de Química, Universidad Nacional Autónoma de México, Circuito Exterior, Ciudad Universitaria, \\ Coyoacan 04510, México, D.F. México
}

(Received June 11, 2001; Accepted February 12, 2002)

\begin{abstract}
A variety of synthetic approaches to highly substituted aromatic natural products is an area of our interest. ${ }^{1}$ The methyl ester of the 3-formyl-4,6-dihydroxy-5-isopropyl-2-methylbenzoic acid, 1, which is a synthetical intermediate to the Espintanol, ${ }^{2}$ was synthesized in our laboratory, and upon crystallization was obtained as colorless crystals. Its structure was determined by an X-ray crystal analysis. Data were collected on a Siemens P4 diffractometer. Crystallographic data as well as details of an Xray data collection and a structure refinement are summarized in Table 1. The intensities were collected using nickel-filtered $\mathrm{Cu}$ $\mathrm{K}_{\alpha}$ radiation $(\lambda=1.54178 \AA)$ at room temperature, and were corrected for Lorentz and polarization effects. ${ }^{3}$ The structure was solved by direct methods ${ }^{4}$ and refined by full-matrix leastsquares with anisotropic temperature factors for the nonhydrogen atoms. The structure solution and refinement
\end{abstract}

Table 1 Crystallographic Data for 1

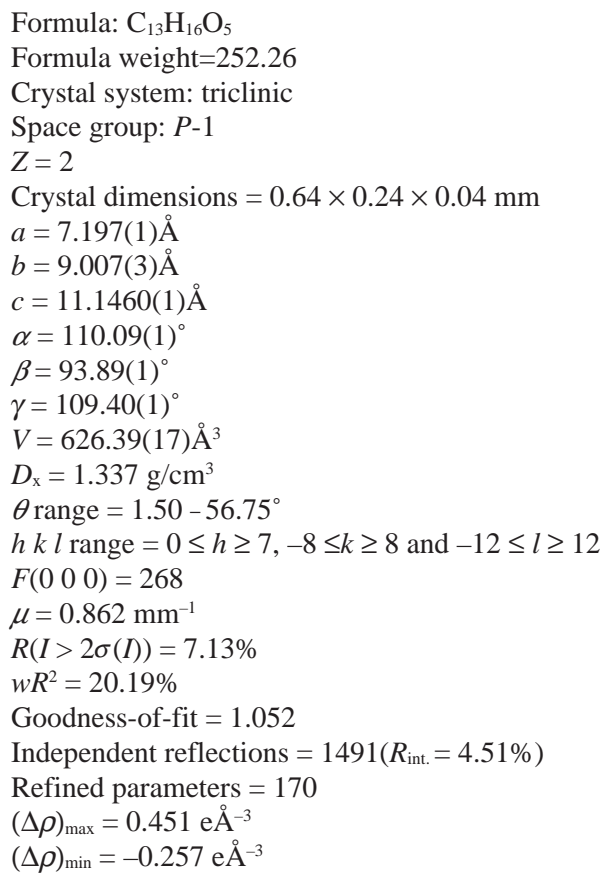

† To whom correspondence should be addressed. included all unique reflections. The hydroxyl H-atoms were located on a different Fourier map, and their positional parameters were refined.

A diagram of the structure of compound $\mathbf{1}$ is shown in Fig. 1. The molecular structure along with the atomic numbering is shown in Fig. 2. The bond lengths and angles agree with the expected values. The molecule is essentially planar with its mean plane bisecting the bond angle of the isopropyl group. Two strong intramolecular hydrogen bonds fix the carbonyl groups orientation $[\mathrm{H} 5 \cdots \mathrm{O} 1,1.72(6) \AA$; O5 $\cdots \mathrm{O} 1,2.534(4) \AA$,

Table 2 Atomic coordinates $\left(\times 10^{4}\right)$ and equivalent isotropic displacement coefficients $\left(\AA^{2} \times 10^{3}\right)$

\begin{tabular}{lclll}
\hline & $x$ & $y$ & $z$ & $U_{\text {eq }}$ \\
\hline $\mathrm{O}(1)$ & $1809(7)$ & $1209(4)$ & $-460(3)$ & $97(1)$ \\
$\mathrm{O}(2)$ & $1122(5)$ & $2117(4)$ & $-1953(3)$ & $76(1)$ \\
$\mathrm{O}(3)$ & $4078(6)$ & $10174(4)$ & $1561(3)$ & $86(1)$ \\
$\mathrm{O}(4)$ & $3943(5)$ & $8876(4)$ & $3244(3)$ & $67(1)$ \\
$\mathrm{O}(5)$ & $2147(6)$ & $3019(4)$ & $1918(3)$ & $79(1)$ \\
$\mathrm{C}(1)$ & $2292(5)$ & $4150(5)$ & $228(3)$ & $49(1)$ \\
$\mathrm{C}(2)$ & $2676(5)$ & $5576(5)$ & $-77(4)$ & $49(1)$ \\
$\mathrm{C}(3)$ & $3208(6)$ & $7191(5)$ & $943(4)$ & $48(1)$ \\
$\mathrm{C}(4)$ & $3377(6)$ & $7332(5)$ & $2264(4)$ & $50(1)$ \\
$\mathrm{C}(5)$ & $2991(6)$ & $5929(5)$ & $2599(3)$ & $50(1)$ \\
$\mathrm{C}(6)$ & $2472(6)$ & $4354(5)$ & $1572(4)$ & $52(1)$ \\
$\mathrm{C}(7)$ & $1727(6)$ & $2377(6)$ & $-731(4)$ & $56(1)$ \\
$\mathrm{C}(8)$ & $2650(7)$ & $5508(6)$ & $-1456(4)$ & $65(1)$ \\
$\mathrm{C}(9)$ & $3539(8)$ & $8730(6)$ & $705(4)$ & $70(1)$ \\
$\mathrm{C}(10)$ & $3190(8)$ & $6092(5)$ & $4015(4)$ & $66(1)$ \\
$\mathrm{C}(11)$ & $5395(9)$ & $6993(7)$ & $4740(4)$ & $83(2)$ \\
$\mathrm{C}(12)$ & $1846(9)$ & $6929(7)$ & $4752(5)$ & $86(2)$ \\
$\mathrm{C}(13)$ & $630(9)$ & $412(6)-$ & $2918(5)$ & $86(2)$ \\
\hline
\end{tabular}

$U_{\text {eq }}$ defined as one third of the trace of the orthogonalized $U_{\mathrm{ij}}$ tensor.

Table 3 Bond lengths $(\AA)$

\begin{tabular}{llll}
\hline $\mathrm{O}(1)-\mathrm{C}(7)$ & $1.208(6)$ & $\mathrm{O}(2)-\mathrm{C}(7)$ & $1.316(5)$ \\
$\mathrm{O}(2)-\mathrm{C}(13)$ & $1.447(5)$ & $\mathrm{O}(3)-\mathrm{C}(9)$ & $1.231(6)$ \\
$\mathrm{O}(4)-\mathrm{C}(4)$ & $1.346(5)$ & $\mathrm{O}(5)-\mathrm{C}(6)$ & $1.340(5)$ \\
$\mathrm{C}(1)-\mathrm{C}(2)$ & $1.384(6)$ & $\mathrm{C}(1)-\mathrm{C}(6)$ & $1.436(6)$ \\
$\mathrm{C}(1)-\mathrm{C}(7)$ & $1.482(6)$ & $\mathrm{C}(2)-\mathrm{C}(3)$ & $1.411(5)$ \\
$\mathrm{C}(2)-\mathrm{C}(8)$ & $1.516(6)$ & $\mathrm{C}(3)-\mathrm{C}(4)$ & $1.427(6)$ \\
$\mathrm{C}(3)-\mathrm{C}(9)$ & $1.447(7)$ & $\mathrm{C}(4)-\mathrm{C}(5)$ & $1.385(6)$ \\
$\mathrm{C}(5)-\mathrm{C}(6)$ & $1.391(5)$ & $\mathrm{C}(5)-\mathrm{C}(10)$ & $1.526(6)$ \\
$\mathrm{C}(10)-\mathrm{C}(12)$ & $1.532(7)$ & $\mathrm{C}(10)-\mathrm{C}(11)$ & $1.533(7)$ \\
\hline
\end{tabular}


Table 4 Bond angles $\left({ }^{\circ}\right)$

\begin{tabular}{llll}
\hline $\mathrm{C}(7)-\mathrm{O}(2)-\mathrm{C}(13)$ & $116.9(4)$ & $\mathrm{C}(2)-\mathrm{C}(1)-\mathrm{C}(6)$ & $119.3(3)$ \\
$\mathrm{C}(2)-\mathrm{C}(1)-\mathrm{C}(7)$ & $125.3(4)$ & $\mathrm{C}(6)-\mathrm{C}(1)-\mathrm{C}(7)$ & $115.4(4)$ \\
$\mathrm{C}(1)-\mathrm{C}(2)-\mathrm{C}(3)$ & $119.0(4)$ & $\mathrm{C}(1)-\mathrm{C}(2)-\mathrm{C}(8)$ & $123.3(4)$ \\
$\mathrm{C}(3)-\mathrm{C}(2)-\mathrm{C}(8)$ & $117.7(4)$ & $\mathrm{C}(2)-\mathrm{C}(3)-\mathrm{C}(4)$ & $119.7(4)$ \\
$\mathrm{C}(2)-\mathrm{C}(3)-\mathrm{C}(9)$ & $122.4(4)$ & $\mathrm{C}(4)-\mathrm{C}(3)-\mathrm{C}(9)$ & $117.8(3)$ \\
$\mathrm{O}(4)-\mathrm{C}(4)-\mathrm{C}(5)$ & $117.4(4)$ & $\mathrm{O}(4)-\mathrm{C}(4)-\mathrm{C}(3)$ & $120.0(4)$ \\
$\mathrm{C}(5)-\mathrm{C}(4)-\mathrm{C}(3)$ & $122.6(3)$ & $\mathrm{C}(4)-\mathrm{C}(5)-\mathrm{C}(6)$ & $116.3(4)$ \\
$\mathrm{C}(4)-\mathrm{C}(5)-\mathrm{C}(10)$ & $122.3(3)$ & $\mathrm{C}(6)-\mathrm{C}(5)-\mathrm{C}(10)$ & $121.3(4)$ \\
$\mathrm{O}(5)-\mathrm{C}(6)-\mathrm{C}(5)$ & $115.4(4)$ & $\mathrm{O}(5)-\mathrm{C}(6)-\mathrm{C}(1)$ & $121.6(3)$ \\
$\mathrm{C}(5)-\mathrm{C}(6)-\mathrm{C}(1)$ & $123.0(4)$ & $\mathrm{O}(1)-\mathrm{C}(7)-\mathrm{O}(2)$ & $119.9(4)$ \\
$\mathrm{O}(1)-\mathrm{C}(7)-\mathrm{C}(1)$ & $124.5(4)$ & $\mathrm{O}(2)-\mathrm{C}(7)-\mathrm{C}(1)$ & $115.6(4)$ \\
$\mathrm{O}(3)-\mathrm{C}(9)-\mathrm{C}(3)$ & $124.8(4)$ & $\mathrm{C}(5)-\mathrm{C}(10)-\mathrm{C}(12)$ & $113.3(4)$ \\
$\mathrm{C}(5)-\mathrm{C}(10)-\mathrm{C}(11)$ & $111.2(4)$ & $\mathrm{C}(12)-\mathrm{C}(10)-\mathrm{C}(11)$ & $111.7(4)$ \\
$\mathrm{H}(4)-\mathrm{O}(4)-\mathrm{C}(4)$ & $107(4)$ & $\mathrm{H}(5)-\mathrm{O}(5)-\mathrm{C}(6)$ & $101(5)$ \\
\hline
\end{tabular}

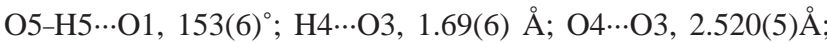
$\left.\mathrm{O} 4-\mathrm{H} 4 \cdots \mathrm{O} 3,152(5)^{\circ}\right]$. In the crystal the molecules stack along the $a$-axis direction with alternant orientation and a mean plane separation of $3.61 \AA$.

\section{Acknowledgements}

This research was supported by a Grant-in-Aid for Scientific Research (No. 27610-E) from CONACyT, México.

\section{References}

1. A. Covarrubias-Zúñiga, N. Zúñiga-Villarreal, and G. Espinosa-Pérez, Anal. Sci., 1998, 14, 865.

2. D. J. Wadsworth and S. Losch, Tetrahedron, 1994, 50, 8673 .<smiles>COC(=O)c1c(C)c(C=O)c(O)c(C(C)C)c1O</smiles>

Fig. 1 Chemical structure of 1

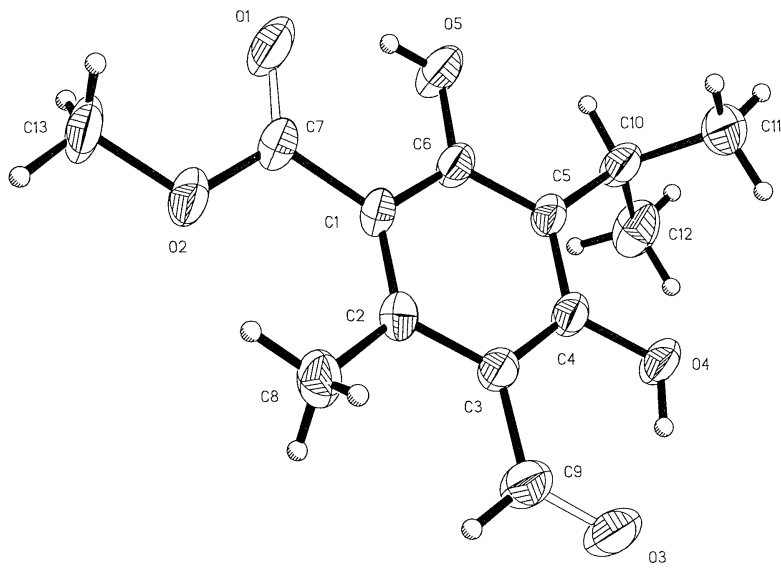

Fig. 2 Plot of $\mathbf{1}$ showing the atom numbering scheme.

3. G. M. Sheldrick, SHELXTL/PC User's Manual, 1990, Siemens Analytical X-ray Instruments Inc., Madison, WI.

4. Siemens XSCANS User's Manual, 1994, Version 2.1, Siemens Analytical X-ray Instruments Inc., Madison. WI. 